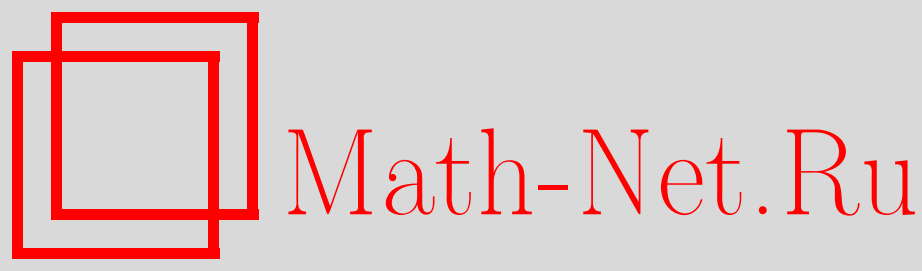

В. В. Борзов, Е. В. Дамаскинский, Инвариантность обобщенного осциллятора относительно линейного преобразования соответствующей системы ортогональных полиномов, ТМФ, 2017, том 190, номер 2, 267-276

DOI: https://doi.org/10.4213/tmf9118

Использование Общероссийского математического портала Math-Net.Ru подразумевает, что вы прочитали и согласны с пользовательским соглашением http://www.mathnet.ru/rus/agreement

Параметры загрузки:

IP : 54.198 .67 .100

26 апреля 2023 г., 15:51:53

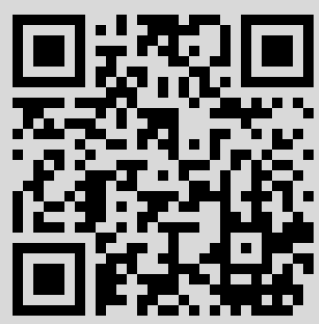




\title{
ФИЗИКА
}

Том 190, № 2

февраль, 2017

\author{
(C) 2017 г. $\quad$ В. В. Борзов*, Е. В. Дамаскинский ${ }^{\dagger}$
}

\section{ИНВАРИАНТНОСТЬ ОБОБЩЕННОГО ОСЦИЛЛЯТОРА ОТНОСИТЕЛЬНО ЛИНЕЙНОГО ПРЕОБРАЗОВАНИЯ СООТВЕТСТВУЮЩЕЙ СИСТЕМЫ ОРТОГОНАЛЬНЫХ ПОЛИНОМОВ}

\begin{abstract}
Рассматриваются семейства многочленов $\mathbb{P}=\left\{P_{n}(x)\right\}_{n=0}^{\infty}$ и $\mathbb{Q}=\left\{Q_{n}(x)\right\}_{n=0}^{\infty}$, ортогональных на вещественной прямой относительно вероятностных мер $\mu$ и $\nu$ соответственно. Предполагается, что многочлены $\left\{Q_{n}(x)\right\}_{n=0}^{\infty}$ и $\left\{P_{n}(x)\right\}_{n=0}^{\infty}$ связаны линейным соотношением. В случае $k=2$ описаны все пары $(\mathbb{P}, \mathbb{Q})$, для которых совпадают алгебры $\mathfrak{A}_{P}$ и $\mathfrak{A}_{Q}$ обобщенных осцилляторов, порождаемые $\left\{Q_{n}(x)\right\}_{n=0}^{\infty}$ и $\left\{P_{n}(x)\right\}_{n=0}^{\infty}$. Построены алгебры обобщенных осцилляторов, соответствующие парам $(\mathbb{P}, \mathbb{Q})$ для произвольного $k \geqslant 1$.
\end{abstract}

Ключевые слова: обобщенные осцилляторы, ортогональные многочлены.

DOI: $10.4213 / \operatorname{tmf} 9118$

\section{1. ВВЕДЕНИЕ}

Пусть $\mathbb{P}=\left\{P_{n}(x)\right\}_{n=0}^{\infty}-$ некоторое семейство полиномов ${ }^{1)}$, ортогональных на вещественной прямой относительно вероятностной меры $\mu$. Рассмотрим последовательность полиномов $\mathbb{Q}=\left\{Q_{n}(x)\right\}_{n=0}^{\infty}$ таких, что

$$
Q_{n}(x)=P_{n}(x)+a_{1} P_{n-1}(x)+\cdots+a_{k} P_{n-k}(x), \quad n>k .
$$

Семейства ортогональных полиномов, связанных таким линейным соотношением, обсуждались в ряде работ (см., например, [1]-[5]). В статье [5] даны необходимые и достаточные условия ортогональности последовательности $\left\{Q_{n}(x)\right\}_{n=0}^{\infty}$ относительно положительной борелевской меры $\nu$ на вещественной прямой.

Е.В. Дамаскинский признателен РФФИ за финансовую поддержку (грант № 15-01-03148_a).

1) Для определенности здесь и далее рассматриваются многочлены с единичным старшим коэффициентом.

*Санкт-Петербургский государственный университет телекоммуникаций, Санкт-Петербург, Россия. E-mail: borzov.vadim@yandex.ru

${ }^{\dagger}$ Военный институт (инженерно-технический) Военной академии материально-технического обеспечения, Санкт-Петербург, Россия. E-mail: evd@pdmi.ras.ru 
Известно [6], что любая последовательность многочленов $\left\{P_{n}(x)\right\}_{n=0}^{\infty}$, ортогональных на вещественной прямой относительно положительной борелевской меры $\mu$, порождает алгебру обобщенного осциллятора $\mathfrak{A}_{P}$. В настоящей работе исследуется вопрос, при каких условиях алгебры $\mathfrak{A}_{P}$ и $\mathfrak{A}_{Q}$, порождаемые такими линейно-связанными полиномами, совпадают, $\mathfrak{A}_{P}=\mathfrak{A}_{Q}$. Эта задача в простейшем случае $k=1$ рассматривалась в работе [7]. В настоящей работе мы обсудим случай $k=2$.

Нам потребуются приводимые ниже результаты из [5], [6], [8]. Пусть $u$ - линейный функционал на линейном пространстве $\mathfrak{P}$ многочленов с вещественными коэффициентами. Многочлены $\left\{P_{n}(x)\right\}_{n=0}^{\infty}$ называются ортогональными относительно $u$, если

$$
\left\langle u, P_{n} P_{m}\right\rangle=0 \quad \forall n \neq m
$$

и

$$
\left\langle u, P_{n}^{2}\right\rangle \neq 0 \quad \forall n=0,1, \ldots
$$

Пусть $H=\left\{u_{i+j}\right\}_{i, j \geqslant 0}$ - матрица Ганкеля, связанная с функционалом $u$, где $u_{k}=\left\langle u, x^{k}\right\rangle, k \geqslant 0$. Линейный функционал $u$ называется квазиопределенным (положительно-определенным), если ведущие подматрицы $H_{n}$ матрицы $H$ несингулярны (положительно-определены) при всех $n \geqslant 0$.

Теорема Фавара дает описание квазиопределенного (положительно-определенного) линейного функционала в терминах трехчленного рекуррентного соотношения, которому удовлетворяют многочлены $\left\{P_{n}(x)\right\}_{n=0}^{\infty}$ :

$$
\begin{gathered}
x P_{n}(x)=P_{n+1}(x)+\beta_{n} P_{n}(x)+\gamma_{n} P_{n-1}(x), \\
P_{0}(x)=1, \quad P_{1}(x)=x-\beta_{0},
\end{gathered}
$$

при $\gamma_{n} \neq 0$ (соответственно, $\left.\gamma_{n}>0\right)$.

Если $u$ - положительно-определенный линейный функционал, то существует положительная борелевская мера $\mu$ (с носителем на бесконечном подмножестве в $\mathbb{R}$ ) такая, что

$$
\langle u, q\rangle=\int_{\mathbb{R}} q d \mu \quad \forall q \in \mathfrak{P} .
$$

В дальнейшем мы будем использовать следующую теорему.

ТЕОрема 1 [5]. Пусть $\left\{P_{n}(x)\right\}_{n=0}^{\infty}-$ последовательность ортогональных полиномов с рекуррентными соотношениями

$$
\begin{gathered}
x P_{n}(x)=P_{n+1}(x)+\beta_{n} P_{n}(x)+\gamma_{n} P_{n-1}(x), \quad \gamma_{n} \neq 0, \\
P_{0}(x)=1, \quad P_{1}(x)=x-\beta_{0} .
\end{gathered}
$$

Пусть $a_{1}$ и $a_{2}$ - вещественные числа такие, что $a_{2} \neq 0, a Q_{n}(x)$ - полиномы, определяемые соотношениями

$$
Q_{n}(x)=P_{n}(x)+a_{1} P_{n-1}(x)+a_{2} P_{n-2}(x), \quad n \geqslant 3 .
$$

Тогда ортогональность последовательности $\left\{Q_{n}(x)\right\}_{n=0}^{\infty}$ зависит от выбора коэбфичиентов $a_{1}$ и $a_{2}$. Точнее говоря, $\left\{Q_{n}(x)\right\}_{n=0}^{\infty}$ является семейством ортогональных многочленов с рекуррентными соотношениями

$$
x Q_{n}(x)=Q_{n+1}(x)+\widetilde{\beta}_{n} Q_{n}(x)+\widetilde{\gamma}_{n} Q_{n-1}(x), \quad n \geqslant 1, \quad \widetilde{\gamma}_{n} \neq 0,
$$


тогда и только тогда, когда $\gamma_{3}+a_{1}\left(\beta_{2}-\beta_{3}\right) \neq 0$ и выполнено одно из следующих условий:

1) если $a_{1}=0$, то при $n \geqslant 4$ имеем $\beta_{n}=\beta_{n-2}, \gamma_{n}=\gamma_{n-2}$;

2) если $a_{1} \neq 0$ и $a_{1}^{2}=4 a_{2}$, то для $n \geqslant 2$

$$
\beta_{n}=A+B n+C n^{2}, \quad \gamma_{n}=D+E n+F n^{2},
$$

где $a_{1} C=2 F, a_{1} B=2 E-2 F$ u $A, B, C, D, E, F \in \mathbb{R}$;

3) если $a_{1} \neq 0$ и $a_{1}^{2}>4 a_{2}$, то для $n \geqslant 2$

$$
\beta_{n}=A+B \lambda^{n}+C \lambda^{-n}, \quad \gamma_{n}=D+E \lambda^{n}+F \lambda^{-n}
$$

при $a_{1} C=(1+\lambda) F, a_{1} \lambda B=(1+\lambda) E$ и $A, B, C, D, E, F \in \mathbb{R}$, где $\lambda$ - единственное решение на интервале $(-1,1)$ уравнения

$$
a_{1}^{2} \lambda=a_{2}(1+\lambda)^{2}
$$

4) если $a_{1} \neq 0$ и $a_{1}^{2}<4 a_{2}$, a $\lambda=e^{i \theta}, \theta \in(0, \pi)$, - единственное решение уравнения $a_{1}^{2} \lambda=a_{2}(1+\lambda)^{2}$, mo при $n \geqslant 2$

$$
\beta_{n}=A+B e^{i n \theta}+\bar{B} e^{-i n \theta}, \quad \gamma_{n}=D+E e^{i n \theta}+\bar{E} e^{-i n \theta},
$$

где $a_{1} \lambda B=(1+\lambda) E$ и $A, D \in \mathbb{R}, B, F \in \mathbb{C}$.

Приведем определение обобщенного осциллятора, связанного с семейством ортогональных полиномов [6]. Пусть $\mu$ - вероятностная мера на $\mathbb{R}$ с конечными моментами любого порядка

$$
\mu_{n}=\int_{-\infty}^{+\infty} x^{n} d \mu<\infty, \quad n \geqslant 0 .
$$

Эти моменты однозначно определяют две последовательности действительных чисел $\left\{a_{n}\right\}_{n=0}^{\infty}$ и $\left\{b_{n}\right\}_{n=0}^{\infty}$, а также систему ортогональных многочленов $\left\{\Psi_{n}(x)\right\}_{n=0}^{\infty}$ с рекуррентными соотношениями

$$
x \Psi_{n}(x)=b_{n} \Psi_{n+1}(x)+a_{n} \Psi_{n}(x)+b_{n-1} \Psi_{n-1}(x), \quad n \geqslant 0,
$$

и начальными условиями

$$
\Psi_{0}(x)=1, \quad \Psi_{1}(x)=\frac{x-a_{0}}{b_{0}} .
$$

Эти многочлены образуют ортонормированный базис в гильбертовом пространстве $\mathcal{H}=\mathrm{L}_{2}(\mathbb{R} ; \mu)$.

Следует различать два случая:

1) $a_{n}=0$ - симметричный случай;

2) $a_{n} \neq 0$ - несимметричный случай.

В гильбертовом пространстве $\mathcal{H}$ определим лестничные операторы $a^{ \pm}$и нумерующий оператор $N$ формулами

$$
\begin{aligned}
a^{+} \Psi_{n}(x) & =\sqrt{2} b_{n} \Psi_{n+1}(x), \\
a^{-} \Psi_{n}(x) & =\sqrt{2} b_{n-1} \Psi_{n-1}(x), \\
N \Psi_{n}(x) & =n \Psi_{n}(x), \quad n \geqslant 0 .
\end{aligned}
$$


Пусть $B(N)$ - операторнозначная функция такая, что

$$
\begin{aligned}
B(N) \Psi_{n}(x) & =b_{n-1}^{2} \Psi_{n}(x), \\
B(N+\mathbb{I}) \Psi_{n}(x) & =b_{n}^{2} \Psi_{n}(x), \quad n \geqslant 0 .
\end{aligned}
$$

Справедлива следующая теорема.

Теорема 2 [6]. Операторы $a^{ \pm}, N, \mathbb{I}$ удовлетворяют соотношениям

$$
a^{-} a^{+}=2 B(N+\mathbb{I}), \quad a^{+} a^{-}=2 B(N), \quad\left[N, a^{ \pm}\right]= \pm a^{ \pm} .
$$

ОпРЕДЕлЕниЕ 1. Ассоциативная алгебра $\mathfrak{A}_{\Psi}$, порождаемая операторами $a^{ \pm}, N$ и II, удовлетворяющими соотношениям (5), а также коммутаторами этих операторов, называется алгеброй обобщенного осииллятора, соответствующей системе ортонормированных многочленов $\left\{\Psi_{n}(x)\right\}_{n=0}^{\infty}$, с рекуррентными соотношениями (4).

Приведем одно полезное следствие теоремы 2. Пусть $\mathfrak{A}_{s}$ - алгебра обобщенного осциллятора, соответствующая рекуррентным соотношениям (4) в симметричном случае $\left(a_{n}=0\right)$, а $\mathfrak{A}_{a}$ - алгебра обобщенного осциллятора, соответствующая рекуррентным соотношениям (4) в несимметричном случае $\left(a_{n} \neq 0\right)$. Тогда $\mathfrak{A}_{s}=\mathfrak{A}_{a}$.

Теперь мы готовы сформулировать рассматриваемую задачу. Пусть даны два семейства многочленов $\mathbb{P}=\left\{P_{n}(x)\right\}_{n=0}^{\infty}$ и $\mathbb{Q}=\left\{Q_{n}(x)\right\}_{n=0}^{\infty}$, ортогональных относительно вероятностных мер $\mu$ и $\nu$ соответственно. Будем предполагать, что эти полиномы удовлетворяют условиям теоремы 1 , причем $\beta_{n}, \widetilde{\beta}_{n}, \gamma_{n}, \widetilde{\gamma}_{n} \in \mathbb{R}$. Обозначим через $\mathfrak{A}_{P}$ и $\mathfrak{A}_{Q}$ соответствующие алгебры обобщенных осцилляторов.

ЗАДАчА. Требуется описать все пары семейств ортогоналъных полиномов $(\mathbb{P}, \mathbb{Q})$, для которьх $\mathfrak{A}_{P}=\mathfrak{A}_{Q}$.

\section{2. МАТРИЦЫ ЯКОБИ И ОСНОВНОЙ РЕЗУЛЬТАТ}

Пусть $\mathbb{P}$ и $\mathbb{Q}$ определены в гильбертовых пространствах $\mathcal{H}_{\mu}=\mathrm{L}^{2}(\mathbb{R} ; \mu)$ и $\mathcal{H}_{\nu}=$ $\mathrm{L}^{2}(\mathbb{R} ; \nu)$ соответственно. Пусть эти полиномы удовлетворяют рекуррентным соотношениям (1) и (3) соответственно. Кроме того, будем считать, что эти многочлены связаны соотношением (2). Мы будем предполагать также, что $\beta_{n}, \widetilde{\beta}_{n}, \gamma_{n}, \widetilde{\gamma}_{n} \in \mathbb{R}$ и что коэффициенты $\beta_{n}, \gamma_{n}$ удовлетворяют условиям теоремы 1.

Матрицы Якоби $J_{P}, J_{Q}$, соответствующие рекуррентным соотношениям (1), (3), могут быть записаны в виде

$$
J=\left[\begin{array}{cc}
A & I_{1} \\
I_{2} & B
\end{array}\right], \quad I_{1}=\left[\begin{array}{cccc}
0 & 0 & 0 & \cdots \\
0 & 0 & 0 & \cdots \\
1 & 0 & 0 & \ldots
\end{array}\right], \quad I_{2}=\left[\begin{array}{ccc}
0 & 0 & \gamma_{3} \\
0 & 0 & 0 \\
0 & 0 & 0 \\
\vdots & \vdots & \vdots
\end{array}\right]
$$

где матрицы $A$ для многочленов $\mathbb{P}$ и $\mathbb{Q}$ имеют вид

$$
A_{P}=\left[\begin{array}{ccc}
\beta_{0} & 1 & 0 \\
\gamma_{1} & \beta_{1} & 1 \\
0 & \gamma_{2} & \beta_{2}
\end{array}\right], \quad A_{Q}=\left[\begin{array}{ccc}
\widetilde{\beta}_{0} & 1 & 0 \\
\widetilde{\gamma}_{1} & \widetilde{\beta}_{1} & 1 \\
0 & \widetilde{\gamma}_{2} & \widetilde{\beta}_{2}
\end{array}\right]
$$


а матрица $B$ равна

$$
B=\left[\begin{array}{ccccccc}
\beta_{3} & 1 & 0 & 0 & 0 & 0 & \ldots \\
\gamma_{2} & \beta_{2} & 1 & 0 & 0 & 0 & \ldots \\
0 & \gamma_{3} & \beta_{3} & 1 & 0 & 0 & \ldots \\
0 & 0 & \gamma_{2} & \beta_{2} & 1 & 0 & \ldots \\
\vdots & \vdots & \ddots & \ddots & \ddots & \ddots & \ddots
\end{array}\right]
$$

Отметим, что элементы $\left(\beta_{0}, \beta_{1}, \beta_{2}, \beta_{3}\right)$ и $\left(\gamma_{1}, \gamma_{2}, \gamma_{3}\right)$ в матрице $J_{P}$ заданы, а элементы $\left(\widetilde{\beta}_{0}, \widetilde{\beta}_{1}, \widetilde{\beta}_{2}\right)$ и коэффициенты $a_{1}, a_{2}$ уравнения $(2)$ должны быть определены.

Мы рассмотрим все четыре случая теоремы 1 . Общие соотношения, справедливые для всех случаев, имеют вид

$$
\begin{aligned}
& \widetilde{\gamma}_{n}=\gamma_{n}, \quad n \geqslant 1 ; \quad \widetilde{\beta}_{n}=\beta_{n}, \quad n \geqslant 3 ; \\
& \beta_{2 n+1}=\beta_{3}, \quad n \geqslant 1 ; \quad \gamma_{2 n}=\gamma_{2}, \quad n \geqslant 1 \text {; } \\
& \beta_{2 n}=\beta_{2}, \quad n \geqslant 1 ; \quad \gamma_{2 n+1}=\gamma_{3}, \quad n \geqslant 1 \text {; } \\
& \gamma_{n} \neq 0 ; \quad a_{2} \neq 0 \text {. }
\end{aligned}
$$

Далее мы будем использовать следующие обозначения:

$$
\begin{aligned}
s_{1} & =\frac{\gamma_{3}-\gamma_{1}-\left(\beta_{2}-\beta_{1}\right)\left(\beta_{3}-\beta_{1}\right)}{\gamma_{3}}, \\
s_{2} & =\frac{\gamma_{2}}{\beta_{3}-\beta_{1}}, \quad s_{3}=\frac{\gamma_{3}}{\beta_{3}-\beta_{1}}, \\
w & =\frac{a_{1}}{4 s_{3}}-\frac{\gamma_{2}}{\gamma_{3}}, \quad w_{\lambda}=\frac{\lambda}{(1+\lambda)^{2}} \frac{a_{1}}{s_{3}}-\frac{\gamma_{2}}{\gamma_{3}} .
\end{aligned}
$$

В терминах матриц Якоби основной результат состоит в следующем утверждении.

Можно доказать, что все пары систем ортогональных полиномов $\left\{P_{n}(x)\right\}_{n=0}^{\infty}$ и $\left\{Q_{n}(x)\right\}_{n=0}^{\infty}$, связанных линейной зависимостью (2), которые порождают одну и ту же алгебру обобщенного осциллятора, можно разбить на восемь групп.

Случай I: $a_{1}=0, \beta_{1} \neq \beta_{3}$. В этом случае матрица $A_{Q}$ и коэффициент $a_{2}$ однозначно определяются соотношениями

$$
\begin{gathered}
\widetilde{\beta}_{0}=\beta_{0}+\frac{\left(\beta_{3}-\beta_{1}-\beta_{0}\right) \gamma_{1}}{\gamma_{2} \gamma_{3}} a_{2}, \quad a_{2}=-s_{1} s_{3}^{2}, \\
\widetilde{\beta}_{1}=\beta_{1}+\frac{a_{2}}{s_{3}}-\widetilde{\beta}_{0}, \quad \widetilde{\beta}_{2}=-\frac{a_{2}}{s_{3}}, \\
\widetilde{\gamma}_{n}=\gamma_{n}, \quad n \geqslant 1, \quad \widetilde{\beta}_{n}=\beta_{n}, \quad n \geqslant 3 .
\end{gathered}
$$

Случай II: $a_{1}=0, \beta_{1}=\beta_{3}, \gamma_{3}=\gamma_{1}, \beta_{2} \neq \beta_{0}$. В этом случае матрица $A_{Q}$ и коэффициент $a_{2}$ однозначно определяются соотношениями

$$
\begin{gathered}
\widetilde{\beta}_{0}=\beta_{1}-\frac{\gamma_{2}}{\beta_{2}-\beta_{0}}, \quad \widetilde{\beta}_{1}=\beta_{0}+\frac{\gamma_{2}}{\beta_{2}-\beta_{0}}, \\
\widetilde{\beta}_{2}=\beta_{2}, \quad a_{2}=\gamma_{2} \frac{\beta_{1}-\beta_{0}}{\beta_{2}-\beta_{0}}-\frac{\gamma_{2}^{2}}{\left(\beta_{2}-\beta_{0}\right)^{2}}, \\
\widetilde{\gamma}_{n}=\gamma_{n}, \quad n \geqslant 1, \quad \widetilde{\beta}_{n}=\beta_{n}, \quad n \geqslant 3 .
\end{gathered}
$$


Случай III: $a_{1} \neq 0, \beta_{1} \neq \beta_{3}, a_{2}=a_{1}^{2} / 4$. Обозначим через $w$ решение уравнения

$$
16 s_{3}^{2} w^{4}+32 s_{2} s_{3} w^{3}+\left(16 s_{2}^{2}+4 s_{3}^{2}\right) w^{2}+\left(4 s_{2}+s_{1} s_{3}\right) w+s_{1} s_{2}+\frac{\gamma_{2}}{\gamma_{3}}\left(\beta_{2}-\beta_{1}\right)=0
$$

такое, что $a_{1}=\left(4 s_{3} w+4 s_{2}\right) \in \mathbb{R}$, и введем величину

$$
C_{\beta, \gamma}=a_{2}\left[-\frac{\gamma_{1}}{\gamma_{3}}\left(\beta_{2}-a_{1}(w+1)\right)+\beta_{0}\left(4 w^{3}+4 w+1\right)\right]-a_{1} w\left[\beta_{0}\left(\beta_{1}+\beta_{2}\right)+\gamma_{1}\right] .
$$

В этом случае при заданном $w$ матрица $A_{Q}$ и коэффициент $a_{2}$ однозначно определяются соотношениями

$$
\begin{gathered}
\widetilde{\beta}_{0}=\beta_{0}-\frac{C_{\beta, \gamma}}{\gamma_{2}}, \quad \widetilde{\beta}_{1}=\beta_{1}+\frac{C_{\beta, \gamma}}{\gamma_{2}}+a_{1} w, \\
\widetilde{\beta}_{2}=\beta_{2}-a_{1}(w+1), \\
a_{1}=4 s_{3} w+4 s_{2}, \quad a_{2}=\frac{1}{4} a_{1}^{2} .
\end{gathered}
$$

Случай IV: $a_{1} \neq 0, \beta_{1}=\beta_{3}, a_{2}=a_{1}^{2} / 4$. Пусть $a_{1}$ - вещественное решение уравнения

$$
\frac{\gamma_{2}^{2}}{\gamma_{3}^{2}} a_{1}^{2}-a_{1}\left(\frac{\gamma_{2}}{\gamma_{3}}+\frac{\gamma_{1}}{4 \gamma_{3}}-\frac{1}{4}\right)+\frac{\gamma_{2}}{\gamma_{3}}\left(\beta_{2}-\beta_{1}\right)=0 .
$$

В этом случае матрица $A_{Q}$ однозначно определяется соотношениями

$$
\begin{gathered}
\widetilde{\beta}_{0}=\beta_{0}-\frac{D_{\beta, \gamma}}{\gamma_{2}}, \quad \widetilde{\beta}_{1}=\beta_{1}+\frac{D_{\beta, \gamma}}{\gamma_{2}}+a_{1} w, \\
\widetilde{\beta}_{2}=\beta_{2}-a_{1}(w+1),
\end{gathered}
$$

где $w=-\gamma_{2} / \gamma_{3}$ и $D_{\beta, \gamma}$ определяется формулой (10) при $w=-\gamma_{2} / \gamma_{3}$.

Случай V: $a_{1} \neq 0, a_{1}^{2}>4 a_{2}, a_{2}=\left(\lambda /(1+\lambda)^{2}\right) a_{1}^{2}, \beta_{1} \neq \beta_{3}, \lambda \in(-1,1)$. Пусть $w_{\lambda}-$ решение уравнения

$$
\begin{gathered}
\frac{(1+\lambda)^{4}}{\lambda^{2}} s_{3}^{2} w_{\lambda}^{4}+2 \frac{(1+\lambda)^{2}}{\lambda} s_{2} s_{3} w_{\lambda}^{3}+\left(\frac{(1+\lambda)^{4}}{\lambda^{2}} s_{2}^{2}+\frac{(1+\lambda)^{2}}{\lambda} s_{3}\right) w_{\lambda}^{2}+ \\
+\left(\frac{(1+\lambda)^{2}}{\lambda} s_{2}+s_{1} s_{3}\right) w_{\lambda}+s_{1} s_{2}+\frac{\gamma_{2}}{\gamma_{3}}\left(\beta_{2}-\beta_{1}\right)=0
\end{gathered}
$$

такое, что

$$
a_{1}=\frac{(1+\lambda)^{2}}{\lambda}\left(s_{3} w_{\lambda}+s_{2}\right) \in \mathbb{R} .
$$

В этом случае матрица $A_{Q}$ однозначно определяется соотношениями

$$
\widetilde{\beta}_{0}=\beta_{0}-\frac{C_{\lambda}}{\gamma_{2}}, \quad \widetilde{\beta}_{1}=\beta_{1}+\frac{C_{\lambda}}{\gamma_{2}}+a_{1} w_{\lambda}, \quad \widetilde{\beta}_{2}=\beta_{2}-a_{1}\left(w_{\lambda}+1\right),
$$

где

$$
\begin{aligned}
C_{\lambda}= & a_{2}\left[-\frac{\gamma_{1}}{\gamma_{3}}\left(\beta_{2}-a_{1}\left(w_{\lambda}+1\right)\right)+\beta_{0}\left(\frac{(1+\lambda)^{2}}{\lambda}\left(w_{\lambda}^{2}+w_{\lambda}\right)+1\right)\right]- \\
& \quad-a_{1} w_{\lambda}\left[\beta_{0}\left(\beta_{1}+\beta_{2}\right)+\gamma_{1}\right], \\
a_{1}= & \frac{(1+\lambda)^{2}}{\lambda}\left(s_{3} w_{\lambda}+s_{2}\right), \quad a_{2}=\frac{\lambda}{(1+\lambda)^{2}} a_{1}^{2},
\end{aligned}
$$

a $\lambda$ - свободный параметр. 
Случай VI: $a_{1} \neq 0, a_{1}^{2}>4 a_{2}, a_{2}=\left(\lambda /(1+\lambda)^{2}\right) a_{1}^{2}, \beta_{1}=\beta_{3}, \lambda \in(-1,1)$. Пусть $a_{1}$ является решением уравнения

$$
\begin{gathered}
\frac{\gamma_{2}^{2}}{\gamma_{3}^{2}} a_{1}^{2}-a_{1}\left[\frac{\gamma_{2}}{\gamma_{3}}+\frac{\lambda}{(1+\lambda)^{2}}\left(\frac{\gamma_{1}}{\gamma_{3}}-1\right)\right]+\frac{\gamma_{2}}{\gamma_{3}}\left(\beta_{2}-\beta_{1}\right)=0 \\
a_{2}=\frac{\lambda}{(1+\lambda)^{2}} a_{1}^{2}
\end{gathered}
$$

В этом случае матрица $A_{Q}$ однозначно определяется соотношениями

$$
\widetilde{\beta}_{0}=\beta_{0}-\frac{D_{\lambda}}{\gamma_{2}}, \quad \widetilde{\beta}_{1}=\beta_{1}+\frac{D_{\lambda}}{\gamma_{2}}+a_{1} w_{\lambda}, \quad \widetilde{\beta}_{2}=\beta_{2}-a_{1}\left(w_{\lambda}+1\right),
$$

где $w_{\lambda}=-\gamma_{2} / \gamma_{3}$ и $D_{\lambda}$ определяются соотношением (16) для $C_{\lambda}$ с $w_{\lambda}=-\gamma_{2} / \gamma_{3}$.

Случаи VII и VIII: $a_{1} \neq 0, a_{1}^{2}<4 a_{2}, a_{2}=\left(\lambda /(1+\lambda)^{2}\right) a_{1}^{2}, \lambda=e^{i \theta}, \theta \in(0, \pi)$. Остальные соотношения для случая VII совпадают с соотношениями для случая V, а в случае VIII - с аналогичными соотношениями для случая VI.

\section{3. ВОЗМОЖНОСТЬ ОБОБЩЕНИЯ НА СЛУЧАЙ $k>2$}

Приведем несколько замечаний о построении всех возможных пар полиномиальных систем, связанных линейной зависимостью общего вида

$$
Q_{n}(x)=P_{n}(x)+a_{1} P_{n-1}(x)+\cdots+a_{k} P_{n-k}(x), \quad k \geqslant 2,
$$

для которых $\mathfrak{A}_{P}=\mathfrak{A}_{Q}$.

Из результатов работы [5] вытекают следующие утверждения:

1) $\widetilde{\gamma}_{n}=\gamma_{n}, n \geqslant 1$

2) $a_{1}, a_{2}, \ldots, a_{k} \in \mathbb{R}, a_{k} \neq 0, \gamma_{n} \neq 0, n \geqslant 1$;

3) $\widetilde{\beta}_{n}=\beta_{n}, \widetilde{\gamma}_{n}=\gamma_{n}+a_{1}\left(\beta_{n-1}-\beta_{n}\right) \neq 0, n \geqslant k+1$;

4) $\gamma_{n}-\gamma_{n-k}=a_{1}\left(\beta_{n-1}-\beta_{n}\right), n \geqslant k+2$.

Необходимо рассмотреть два случая:
a) $a_{1}=0$
б) $a_{1} \neq 0, \quad \beta_{n}=\beta_{k}$ при $n \geqslant k$.

В обоих случаях имеем $\gamma_{n}=\gamma_{n-k}, n \geqslant k+2$.

Затем следует рассмотреть все возможные случаи, когда некоторые из коэффициентов $a_{1}, a_{2}, \ldots, a_{k-1}$ равны нулю, а все остальные не равны нулю. Напомним, что $a_{k} \neq 0$. Число таких вариантов равно

$$
C_{k-1}^{0}+C_{k-1}^{1}+\cdots+C_{k-1}^{k-1}=2^{k-1} .
$$

\section{4. АЛГЕБРА ОБОБЩЕННОГО ОСЦИЛЛЯТОРА, СООТВЕТСТВУЮЩАЯ ПАРЕ $(\mathbb{P}, \mathbb{Q})$}

Рассмотрим алгебру обобщенного осциллятора $\mathfrak{A}_{P}=\mathfrak{A}_{Q}$, соответствующую рассмотренным выше парам $(\mathbb{P}, \mathbb{Q})$ систем ортогональных полиномов, связанных друг с другом линейным соотношением (18). 
Для построения алгебры обобщенного осциллятора $\mathfrak{A}_{\mathbb{P}}$ в гильбертовом пространстве $\mathcal{H}_{\mu}=\mathrm{L}^{2}(\mathbb{R} ; \mu)$ следует симметризовать рекуррентные соотношения (1) (см. работу [6])

$$
x P_{n}(x)=P_{n+1}(x)+\beta_{n} P_{n}(x)+\gamma_{n} P_{n-1}(x), \quad P_{0}(x)=1,
$$

с $\gamma_{0}=0$. $\mathrm{C}$ этой целью перейдем от системы полиномов $\mathbb{P}$ к системе полиномов $\Phi=\left\{\varphi_{n}(x)\right\}_{n=0}^{\infty}$ по формулам

$$
P_{n}(x)=\alpha_{n} \varphi_{n}(x), \quad \alpha_{0}=1, \quad \alpha_{n}=\sqrt{\gamma_{1}}\left(\gamma_{2} \ldots \gamma_{s}\right)^{m / 2}\left(\gamma_{s+1} \ldots \gamma_{k+1}\right)^{(m-1) / 2},
$$

где $n=(m-1) k+s, m \geqslant 1, s=2, \ldots, k+1$, а $k \geqslant 1-$ фиксированное целое число. Это возможно только в случае, если $\gamma_{1} \neq 0, \ldots, \gamma_{k+1} \neq 0$. Тогда рекуррентные соотношения (19) принимают вид

$$
x \varphi_{n}(x)=b_{n} \varphi_{n+1}(x)+\beta_{n} \varphi_{n}(x)+b_{n-1} \varphi_{n-1}(x),
$$

где

$$
\varphi_{0}=1, \quad b_{-1}=0, \quad b_{n}=\sqrt{\gamma_{n+1}}, \quad n \geqslant 0,
$$

и

$$
\gamma_{n}= \begin{cases}\gamma_{1}, & n=1, \\ \gamma_{s}, & n=k m+s, \quad m \geqslant 0, \quad s=2, \ldots, k+1 .\end{cases}
$$

Определим лестничные операторы $a_{\Phi}^{ \pm}$и нумерующий оператор $\mathbb{N}_{\Phi}$ в $\mathcal{H}_{\mu}$ формулами

$$
\begin{gathered}
a_{\Phi}^{+} \varphi_{n}=\sqrt{2 \gamma_{n+1}} \varphi_{n+1}, \quad a_{\Phi}^{-} \varphi_{n}=\sqrt{2 \gamma_{n}} \varphi_{n-1}, \\
\mathbb{N}_{\Phi} \varphi_{n}=n \varphi_{n}, \quad n \geqslant 0 .
\end{gathered}
$$

Пусть $B_{\Phi}\left(\mathbb{N}_{\Phi}\right)$ - операторнозначная функция, определенная равенствами

$$
\begin{aligned}
B_{\Phi}\left(\mathbb{N}_{\Phi}\right) \varphi_{n} & =\gamma_{n} \varphi_{n}, & & n \geqslant 0, \\
B_{\Phi}\left(\mathbb{N}_{\Phi}+I_{\mu}\right) \varphi_{n} & =\gamma_{n+1} \varphi_{n}, & & n \geqslant 0 .
\end{aligned}
$$

Тогда алгебра обобщенного осциллятора $\mathfrak{A}_{\mathbb{P}}$ порождается операторами $a_{\Phi}^{ \pm}, \mathbb{N}_{\Phi}, I_{\mu}$, удовлетворяющими соотношениям

$$
\begin{gathered}
a_{\Phi}^{-} a_{\Phi}^{+}=2 B_{\Phi}\left(\mathbb{N}_{\Phi}+I_{\mu}\right), \quad a_{\Phi}^{+} a_{\Phi}^{-}=2 B_{\Phi}\left(\mathbb{N}_{\Phi}\right), \\
{\left[\mathbb{N}_{\Phi}, a_{\Phi}^{ \pm}\right]= \pm a_{\Phi}^{ \pm},}
\end{gathered}
$$

а также коммутаторами этих операторов. В этом случае квадратичный гамильтониан

$$
H_{\Phi}=a_{\Phi}^{-} a_{\Phi}^{+}+a_{\Phi}^{+} a_{\Phi}^{-}
$$

является самосопряженным оператором в гильбертовом пространстве $\mathcal{H}_{\mu}$.

Ортонормированные многочлены $\left\{\varphi_{n}(x)\right\}_{n=0}^{\infty}$ являются собственными функциями оператора $H_{\Phi}$. Соответствующие собственные значения равны

$$
\lambda_{0}=2 \gamma_{1}, \quad \lambda_{n}=2\left(\gamma_{n}+\gamma_{n+1}\right), \quad n \geqslant 1 .
$$

В заключение заметим, что так как в нашем случае $b_{n}^{2} \neq\left(c_{0}+c_{2} n\right)(1+n)$ (где $\left.c_{0}, c_{1} \in \mathbb{R}\right)$, то согласно результатам работ [9], [10]

$$
\operatorname{dim} \mathfrak{A}_{P}=\operatorname{dim} \mathfrak{A}_{Q}=\infty .
$$

Отметим также, что представляется интересным изучить связь мер ортогональности $\mu$ и $\nu$ в пространствах $\mathcal{H}_{\mu}$ и $\mathcal{H}_{\nu}$ соответственно. 


\section{5. ЗАКЛЮЧИТЕЛЬНЫЕ ЗАМЕЧАНИЯ}

Основным понятием, используемым в настоящей работе, является понятие обобщенного осциллятора, порожденного семейством полиномов, ортогональных относительно некоторой положительной меры $\mu$ на вещественной оси. Гильбертово пространство $\mathrm{L}_{2}(\mathbb{R} ; \mu)$ с базисом, составленным из упомянутых ортогональных полиномов, является фазовым пространством соответствующего обобщенного осциллятора. Интересным с точки зрения физических приложений является вопрос, при каких условиях обобщенный осциллятор инвариантен относительно линейного преобразования базиса фазового пространства? В настоящей работе дается ответ на этот вопрос. А именно, было построено семейство обобщенных осцилляторов, инвариантных относительно линейных преобразований системы ортогональных полиномов, порождающих этот осциллятор.

Заметим, что, хотя проведенное в настоящей работе исследование носит в основном математический характер, имеется перспектива применения полученных результатов в физике, например в квантовой нелинейной оптике, где концепция деформированного осциллятора хорошо известна (см., например, обзор [11]). В частности, в работе [12] вводится понятие $f$-осциллятора и исследуется физический смысл этого понятия. Обобщенный осциллятор построенного в работе класса (который инвариантен относительно линейного преобразования системы ортогональных полиномов, порождающих этот осциллятор) является примером $f$-осциллятора. Соответствующая функция $f$ имеет вид

$$
f(n)= \begin{cases}0, & n=0, \\ 2 \sqrt{\gamma_{1}}, & n=1, \\ 2 \sqrt{\frac{\gamma_{s}}{k m+s}}, & n=k m+s, \quad m \geqslant 0, \quad s=2,3, \ldots, k+1 .\end{cases}
$$

Было бы интересно исследовать физический смысл осцилляторов, построенных в данной работе, но это, к сожалению, выходит за рамки настоящей работы.

Благодарности. Авторы благодарны рецензенту, который обратил наше внимание на работу [12].

\section{Список литературы}

[1] В.Б. Уваров, "О связи систем полиномов, ортогональных относительно различных функций распределения”, Ж. вычисл. матем. и матем. биз., 9:6 (1969), 1253-1262.

[2] K. H. Kwon, J. H. Lee, F. Marcellan, "Orthogonality of linear combinations of two orthogonal sequences", J. Comput. Appl. Math., 137:1 (2001), 109-122.

[3] E. Berriochoa, A. Cachafiero, J. M. Garcia-Amor, "A characterization of the four Chebyshev orthogonal families", Internat. J. Math. Math. Sci., 2005:13 (2005), 2071-2079.

[4] 3. С. Гриншпун, "Дифференциальные уравнения ортогональных многочленов Бернштейна-Сеге”, Дифферени. уравнения, 26:5 (1990), 769-776.

[5] M. Alfaro, F. Marcellán, A. Peña, M. L. Rezola, "When do linear combinations of orthogonal polynomials yield new sequences of orthogonal polynomials?", J. Comput. Appl. Math., 233:6 (2010), 1146-1452.

[6] V. V. Borzov, "Orthogonal polynomials and generalized oscillator algebras", Integral Transform. Spec. Funct., 12:2 (2001), 115-138. 
[7] V.V. Borzov, E. V. Damaskinsky, "On representations of generalized oscillator for two sequences of linearly related orthogonal polynomials", Proceedings of the International Conference "Days on Diffraction 2015" (St. Petersburg, Russia, 25-29 May, 2015), eds. O. V. Motygin, A. P. Kiselev, P. A. Belov, L. I. Goray, A. Ya. Kazakov, A. S. Kirpichnikova, IEEE, New York, 2015, 58-61.

[8] T.S. Chihara, An Introduction to Orthogonal Polynomials, Mathematics and Its Applications, 13, Gordon and Breach, New York, 1978.

[9] G. Honnouvo, K. Thirulogasanthar, "On the dimensions of the oscillator algebras induced by orthogonal polynomials", J. Math. Phys., 55:9 (2014), 093511, 13 pp.

[10] V. V. Borzov, E. V. Damaskinsky, "On dimensions of oscillator algebras", Proceedings of Days on Difraction 2014 (St. Petersburg, Russia, 26-30, May 2014), eds. O. V. Motygin, A. P. Kiselev, L. I. Goray, A. Ya. Kazakov, A. S. Kirpichnikova, IEEE, New York, 2014, $48-52$.

[11] V. V. Dodonov, "Nonclassical' states in quantum optics: a 'squeezed' review of the first 75 years", J. Opt. B: Quantum Semiclass. Opt., 4:1 (2002), R1-R33.

[12] V. I. Man'ko, G. Marmi, E. C. G. Sudarshan, F. Zaccaria, "f-Oscillators and nonlinear coherent states", Phys. Scr., 55:5 (1997), 528-541. 